\title{
Atypical Infective Endocarditis
}

\author{
Paul R. Gordon, MD, and Shawn A. Allen, MD
}

Background: Although infective endocarditis has changed in the recent past as a result of microbiologic and risk factors, it continues to be clinically challenging. The disease is characterized by the formation of septic masses of platelets on the surfaces of heart valves. Several mechanisms can cause or contribute to the development of endocarditis. Although risk factors for infective endocarditis are well known, patients with atypical signs and symptoms continue to challenge us.

Metbods: We describe a case report of a patient admitted to our inpatient service with back pain and presumed pyelonephritis. A MEDLINE literature search was conducted, using the key words "endocarditis," "back pain," and "bacterial," for the years 1986 to the present.

Results and Conclusions: A 42 -year-old woman with a history of intravenous drug abuse was admitted to the family practice service with back pain and pyelonephritis. She developed hypoxia and a new heart murmur and had continued fevers. Blood cultures drawn in the emergency department grew methicillin-resistant Staphylococcus aureus. A bone scan and magnetic resonance imaging led to the diagnosis of epidural abscess. What appeared to be a simple case of pyelonephritis with back pain became a case of infective endocarditis complicated by an epidural abscess.(J Am Board Fam Pract 1999; 12:391-4.)

Infective endocarditis is known to affect many organ systems, and the initial symptoms or physical examination findings can indicate involvement of any of these organ systems. Common findings include heart murmur, valvular bacterial vegetation, arterial emboli-induced hematuria, conjunctival petechiae, Janeway spots, Osler nodes, fever, and varied musculoskeletal complaints. From $25 \%$ to $44 \%$ of patients with bacterial endocarditis will have musculoskeletal complaints, and for $25 \%$ of patients these symptoms will be the first sign of disease. Nevertheless, physicians do not always think of bacterial endocarditis when encountering a patient with fever and back pain. The following report will describe a somewhat atypical case of bacterial endocarditis.

\section{Methods}

A MEDLINE literature search was conducted, using the key words "endocarditis," "back pain," and "bacterial," for the years 1986 to the present. A report of a patient admitted to the family practice inpatient service describes an unusual case of infec-

Submitted, revised, 16 December 1998.

From the Department of Family and Community Medicine (PRG), and the Family Practice Center (SAA), University of Arizona College of Medicine, Tucson, Ariz. Address reprint requests Paul R. Gordon, MD, Department of Family and Community Medicine, University of Arizona, Tucson, AZ 85724 . tive endocarditis complicated by an epidural abscess with symptoms of severe back pain that led to a presumed diagnosis of pyelonephritis.

\section{Case Report}

A 42-year old woman came to the emergency department complaining of a pain in her buttock. At that time she had a blood pressure of $113 / 71 \mathrm{~mm}$ $\mathrm{Hg}$, a pulse rate of 112 beats per minute, a temperature of $37.1^{\circ} \mathrm{C}$, and respirations of $20 / \mathrm{min}$. In the emergency department laboratory tests included a renal panel, complete blood count, and liver function tests.

While in the emergency department she developed a fever to $40^{\circ} \mathrm{C}$. Urine specimens and blood samples were collected for culture. The urinalysis showed a specific gravity of 1.010 with hematuria $(1+)$ and nitrituria. The microscopic urinalysis revealed 4 red blood cells and 18 white blood cells per high-power field and bacteria $(3+)$. A pregnancy test was negative. The patient was admitted with a tentative diagnosis of pyelonephritis.

When she was admitted to the hospital it was learned that she had fallen on her buttocks 3 days before admission while walking her dog. The patient said that her back pain had gotten progressively worse since that time. Her pain started in her back, went into her left buttock, and radiated down the posterior aspect of her left leg to below her knee. 
The patient's medical history was notable for intravenous heroine use, and she had been on methadone for 10 years. She reported that a blood test had been negative for human immunodeficiency virus 10 months earlier. Her medical history was also notable for a tonsillectomy and a bout of coccidioidomycosis, the details of which were unknown. She was a smoker ( 2 packs per day). Her last use of heroin was 3 days before admission. During a review of symptoms she complained of insomnia and difficulty concentrating and had an undocumented and unintentional $20-\mathrm{lb}$ weight loss in the past month. She denied any fevers, shortness of breath, or chest pain. She had some diarrhea but denied any vomiting. She denied any dysuria but complained of increasing frequency of urination.

When examined, she was a thin, unkempt woman in moderate distress secondary to pain and uncooperative with parts of the examination. Findings of a head, ears, nose, and throat examination were remarkable only for poor dentition. Her upper extremity was covered with multiple tattoos and scars. She had a sinus rhythm at a rate of 112 beats per minute without appreciable murmur, and her lungs were clear bilaterally. There were positive bowel sounds, her abdomen was soft, and there was no hepatosplenomegaly. There was, however, a bilateral flank tenderness to light touch. Her extremities showed no edema, and her pulses $(2+)$ were symmetrical. Neurologically her muscle strength was $4 / 5$ in the lower extremities, with the decrease attributed to pain. Her senses were intact, and she had deep tendon reflexes $(1+)$ bilaterally at the patellar tendon. Psychologically the patient was irate.

Our initial assessment of this patient was that she had a pyelonephritis and possibly sciatica resulting from her fall a few days earlier. We admitted her and prescribed antibiotics empirically (intravenous ceftriaxone). Urine cultures the next morning were positive for Escherichia coli (4+). During the second day of hospitalization the patient was noted to be hypoxic.

Further workup included a chest radiograph, Doppler studies of the lower extremities, and a ventilation-perfusion scan. The radiograph showed no evidence of focal disease. There was no deep vein thrombosis apparent on the Doppler studies. The ventilation-perfusion scan indicated low probability for a pulmonary embolism. A new physical finding on subsequent examination was a grade $2 / 6$ systolic murmur heard best at the left sternal bor $\frac{\mathrm{T}}{3}$ der.

On the second hospital day, the laboratory re $\frac{0}{2}$ ported preliminary results of blood samples col-i lected in the emergency department upon patient's admission as positive for Stapbylococcus. Now we were caring for a 42-year-old woman with a history? of intravenous drug use who continued to haves temperature elevations, had positive blood cultures, needed supplemental oxygen, and had a new sys $\frac{\bar{\sigma}}{\bar{c}}$. tolic murmur. Our suspicion for endocarditis waso high. A transthoracic echocardiogram was orderedฆ It showed mitral and tricuspid regurgitation $(1+) \vec{\circ}$ with no vegetations.

On the third hospital day, blood samples col lected in the emergency department were positive for methicillin-resistant Staphylococcus aureus. Three of the four blood samples cultured within the first 2 days of hospitalization were also positive for me-ie thicillin-resistant Staphylococcus aureus. The patient continued to have pain in her back, which radiated down her left leg and was poorly controlled withes narcotic analgesics.

At admission the patient's back pain was attrib- $-\frac{3}{3}$ uted to a combination of the pyelonephritis and the injury she sustained during her fall. Had that beenco the case, however, we believed her pain should have been abating. She was on appropriate antibiotics for her infections. Nevertheless, her pain continuedo to be poorly controlled. Her picture was compli-命 cated by her history of intravenous drug use and drug-seeking behavior. After a second neurologic 3 examination showed a substantial decrease in strength in the left lower extremity, a bone scan was ordered.

Results of the bone scan showed an increase uptake in the region of the left L5 vertebra. Based on these results, magnetic resonance imaging was? obtained, which showed an epidural abscess with associated edema within the $L 5$ and $S 1$ vertebral bodies.

In summary, what we thought was a simple case of pyelonephritis with back pain became a case of infective endocarditis complicated by an epiduralo abscess.

\section{Discussion}

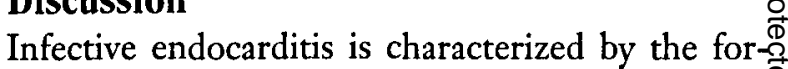
mation of septic masses of platelets on the surfaces $\stackrel{\mathbb{Q}}{\Omega}$ of heart valves. Several mechanisms can cause or 
contribute to the development of endocarditis. Preexisting cardiac disease can cause high-flow jet streams or turbulence. These lesions caused by high-flow jet streams can cause endothelial thickening with subsequent platelet and fibrin thrombus formation. The thrombus acts as a nidus for bacterial growth. In addition, agglutinating antibodies predispose to the seeding of the sterile vegetations by creating clumps of organisms more likely to attach to evolving fibrinous deposits. Specific bacterial adhesion factors can also play an important part. For example, streptococcus organisms species produce a higher degree of aggregation and adhesion to fibrin and platelet thrombi than do such bacteria as $E$ coli. ${ }^{1}$

\section{Epidemiology}

Clinically, two distinct events have caused the face of infective endocarditis to change dramatically in the recent past. The first event is the apparent shift in the microbiology of this disease. In the $1960 \mathrm{~s}$ and 1970 s approximately $70 \%$ to $80 \%$ of cases of infective endocarditis were caused by Streptococcus viridans or an enterococcus. At that time only about $15 \%$ of cases were due to staphylococci, mainly Staphylococcus aureus. In recent studies streptococci still account for most cases but only slightly more than $50 \%$. In contrast, infective endocarditis caused by staphylococci is reported to have increased to $30 \%$. Some of this shift could be explained by reporting bias. For example, it was reported that in one study there was a disparity between the microbiologic causes of infective endocarditis in patients referred to one tertiary care center and the causes in those patients cared for in surrounding community hospitals. The percentage of cases caused by streptococci was higher and the percentage of cases caused by staphylococci was much lower in the community. It was postulated that clinically staphylococcus endocarditis tends to be more acute and fulminant, which could play a part in the greater proportion of referrals to tertiary care centers. ${ }^{2}$ Also, these tertiary care centers are more likely to report their cases in the literature.

The other event that has affected the epidemiology of infective endocarditis in the past 30 years is a change in the at-risk population. The nature of the underlying cardiac valve lesions upon which infective endocarditis develops has changed. Mitral valve prolapse and degenerative or congenital heart disease have replaced rheumatic valvular disease as a cause of the lesions. ${ }^{3}$ Recently infective endocarditis has tended to affect an older patient population, mainly as a result of declines in incidence of acute rheumatic carditis throughout developed countries. Additionally, the upward shift in age has been linked to advances in the field of cardiothoracic surgery. An older population can now undergo prosthetic valve replacement surgery with a decrease in postoperative morbidity. Prosthetic valve recipients are at risk for infective endocarditis.

Additionally, in urban areas intravenous drug users account for a substantial proportion of endocarditis cases. In one study, more than $50 \%$ of patients with evidence of infective endocarditis found by echocardiogram were intravenous drug users. Other studies have documented that approximately $13 \%$ of febrile intravenous drug users at urban emergency departments have infective endocarditis. $^{4}$

\section{History}

Vertebral osteomyelitis is noted to be a complication of infective endocarditis. ${ }^{5}$ As noted, back pain might be the initial complaint in up to $25 \%$ of patients found to have infective endocarditis, although most case reports document severe back pain in only $7 \%$ to $9 \%$ of patients. Generalized musculoskeletal complaints are even more common, including myalgias and sacroiliac, temporomandibular, and cervical joint tenderness. Only one reference reported in the medical literature from 1966 through 1998, however, cited a case of infective endocarditis initially diagnosed as acute epidural abscess. ${ }^{6}$ That patient was found to have Streptococcus pneumoniae in the abscess and in the blood. The pathogenesis of low-back pain in infective endocarditis is not well understood. Vertebral arterial embolization, vertebral septic necrosis, or immune complex deposition is cited as a possibility.

On reviewing this case retrospectively, a few clinical lessons emerge. We should have paid closer attention to the patient's use of intravenous drugs. We too readily accepted pyelonephritis as a primary diagnosis and sciatica related to trauma as a secondary diagnosis to explain the pain in her buttock. We failed to find a unifying diagnosis. Our initial physical examination might not have been sufficient, and a more thorough neurologic and cardiovascular examination might have revealed other clues. We could have too readily relied on 
our initial primary diagnosis of pyelonephritis. In this patient with serious risk factors, our index of suspicion was not high enough.

It is very easy to be critical retrospectively, however. As it is stated, "hindsight is 20/20." Certain aspects of this patient's management were quite appropriate. We immediately recognized her lack of responsiveness to what should have been appropriate antimicrobial medication for a patient with pyelonephritis. We provided adequate follow-up to this lack of responsiveness with repeated physical and laboratory examinations. We continued to question our diagnosis when faced with blood cultures growing Staphylococcus aureus. We looked for a unifying diagnosis of the bacteremia and continued back pain.

The prevalence of infective endocarditis is cited to be around 0.33 per 1000 admissions. If only $7 \%$ to $9 \%$ of these patients have back pain as their chief complaint, nearly 38,000 patients would be admitted to the hospital before there would be a patient with infective endocarditis whose chief complaint was back pain. Infective endocarditis is difficult to $\frac{7}{3}$ diagnose, and when the initial complaint is back ${ }^{\circ}$ pain, it is even more difficult. Infective endocarditis with severe back pain is another diagnosis family physicians need to be aware of, but it is rare.

\section{References}

1. Danilowicz D. Infective endocarditis. Pediatr Rev으 1995;16:148-54.

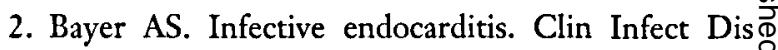
1993;17:313-22.

3. Saccente M, Cobbs CG. Clinical approach to infec- $\vec{\circ}$ tive endocarditis. Cardiol Clin 1996;14:351-62.

4. DeWitt DE, Pauuw DS. Endocarditis in injection N drug users. Am Fam Physician 1996;53:2045-9.

5. Ogata Y, Yabe T, Matsumura Y, Takata J, Chikamori $T$, Doi $Y$. [Infective endocarditis associated $\vec{N}$ with vertebral osteomyelitis: report of two cases.] [Japanese]. J Cardiol 1996;27(Suppl 2):97-101.

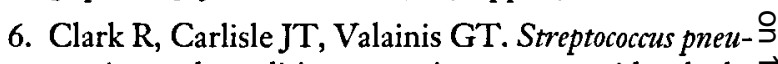
moniae endocarditis presenting as an epidural $a b-\vec{c}$ scess. Rev Infect Dis 1989;11:338-40. 